\title{
VI Jornada de Jóvenes Investigadores del I3A 2 de junio de 2017
}

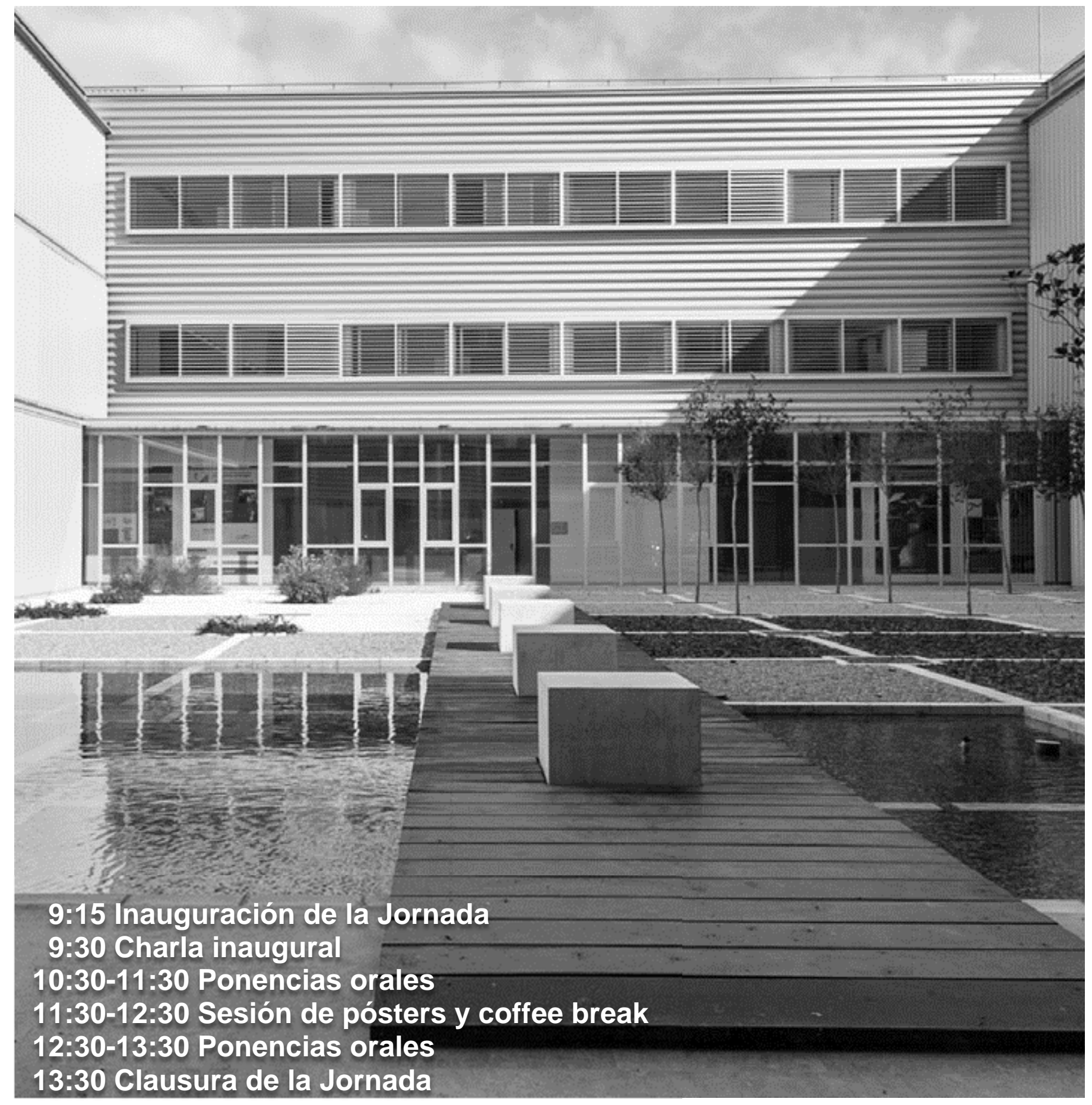

y.\#jovenesI3A 
PROGRAMA VI JORNADA DE JÓVENES INVESTIGADORES DEL I3A

2 de junio de 2017

Sala de Conferencias del Ed. I+D (bloque 1, $1^{\mathrm{a}}$ planta)

\section{9:15 Inauguración}

9:30 Conferencia inaugural a cargo de José Antonio Sanz (Profesor Contratado Doctor de la Universidad de Sevilla y que realizó su doctorado en el I3A). Investigación multidisciplinar como base del profesor universitario joven en Ingeniería

\section{0:30-11:30 Ponencias orales}

10:30 Characterization of SiO2 Nanoparticles by Single Particle- Inductively Coupled PlasmaTandem Mass Spectroscopy

Diego Pereira Leite, Eduardo Bolea-Fernández, Ana Rúa Ibarz, Martín Resano, Frank Vanhaecke, Maite Aramendía

10:45 Simulación 3D de implantes de anillos intraestromales para la estabilización del queratocono

Julio Flecha Lescun, Miguel Ángel Ariza-Gracia, Jesús Zurita, Begoña Calvo

11:00 Control vectorial para motores lineales de una plataforma de nanoposicionamiento Lucía Candela Díaz Pérez, Marta Torralba, José Antonio Albajez, José Antonio Yagüe

11:15 Unmanned Aerial Vehicel Based Wireless Sensor Network for Marine-Coastal Environment Monitoring

Carlos Andrés Trasviña Moreno, Rubén Blasco Marín, Alvaro Marco Marco, Roberto Casas Nebra

11:30-12:30 Sesión de pósters y coffee break (Ed. I+D, planta calle, zona ajardinada)

\section{2:30-13:30 Ponencias orales}

12:30 Obtención de sílice precipitada en un sistema de 2 reactores consecutivos Diego Zapater Bes, Ramón Campo, Jaime Soler, Miguel Menéndez

12:45 Heart Rate Variability Analysis in Risk of Asthma Stratification Javier Milagro, Eduardo Gil, Jesús Lázaro, Ville-Pekka Seppä, L. Pekka Malmberg, Anna S. Pelkonen, Anne Kotaniemi-Syrjänen, Mika J. Makelä, Jari Viik, Raquel Bailón

13:00 Improved Precoder Architecture for Duobinary Transceiver Javier Aguirre Olcoz, C. Sánchez-Azqueta, E. Guerrero, C. Gimeno, S. Celma

13:15 Plataforma para el desarrollo de Entornos Colaborativos Interactivos Clara Bonillo Fernández, Eva Cerezo 


\section{SESIÓN DE PÓSTERS}

\section{INGENIERÍA BIOMÉDICA}

- Tribological Behavior and Mechanical Properties of Graphene/PEEK Composites. J. Morris, R. Ríos, M. Castro, J.A. Puértolas

- Modelo numérico del proceso de reestenosis tras la implantación de un stent. Javier Escuer, Estefanía Peña, Miguel Ángel Martínez

- Assessment of Quadratic Nonlinear Cardiorespiratory Couplings During Tilt Table Test by Means of Real Wavelet Biphas. Spyridon Kontaxis, Jesús Lázaro, Eduardo Gil, Pablo Laguna, Raquel Bailón

- New Structured Materials in the Study of the Mechanobiological Processes Related to the Heart Failure. Sandra González Lana, Andrés Belaza, Alan Vigueras, María Virumbrales, Guillermo Llamazares, Laura Asín, Jesús M. de la Fuente, Francisco Medel, Mohamed Hamdy Doweidar, Sara Oliván, Luis Fernández Ledesma, Manuel Doblaré, Arantxa González Miqueo, Iñaki Ochoa, Carlos Sánchez-Somolinos

- Chemo-mechanical model for skeletal muscle contraction. M. Karami, B. Calvo, H. Zohoor, K. Firoozbakhsh, J. Grasa

\section{PROCESOS Y RECICLADO}

- Carbonato de dimetilo como aditivo a combustible diésel para reducir las emisiones de hollín. Katiuska Alexandrino, Ángela Millera, Rafael Bilbao, María U. Alzueta

- Integrating Renewable Energy Technologies and Thermal Energy Storage to Support Building Trigeneration Systems: A Multicriteria Approac. Eduardo Antonio Pina

- Formación de hidrocarburos aromáticos policíclicos (PAH) y hollín en la pirólisis de DMC y DMM. Fausto Viteri, Ángela Millera, Rafael Bilbao, María U. Alzueta

- Etanol como aditivo para combustibles: estudio de la oxidación a alta presión de sus mezclas con acetileno. Lorena Marrodán, Miguel Fuster, Ángela Millera, Rafael Bilbao, María U. Alzueta

- Estudio cinético del reformado seco de biogás.Daniel Zambrano Juca, Jaime Soler, Javier Herguido, Miguel Menéndez

- Estudio experimental y modelado cinético de la oxidación de H2S y su interacción con NO. Juan M. Colom-Díaz, Jorge Conchello, Ángela Millera, Rafael Bilbao, María U. Alzueta

- Biorreactores de membranas cerámicas para tratamiento de aguas residuales urbanas. Patricia Ugarte, Ana Ramo, Mari Carmen Bordes, Magda Lorente, Elena Zuriaga, Enrique Sanchez, Javier Rubert, José Ángel Peña, Miguel Menéndez

- Producción y purificación de hidrógeno mediante "steam-iron" a partir de biogás enriquecido en metano. Jaime Lachén Mateo, Javier Herguido Huerta, José Ángel Peña Llorente

- Interference-free determination of $\mathrm{Ca}$ in ultrapure water, concentrated acids and hydrogen peroxide using isotope dilution, ICP-MS and $\mathrm{CH} 3 \mathrm{~F}$ as a reaction gas. Raquel $\mathrm{C}$ Machado, Maite Aramendia, Ana Rita A. Nogueira, Martin Resano

- Aplicación de la extracción inducida por rupture de microemulsión en la determinación de $\mathrm{Cu}$, $\mathrm{Ni}$ y $\mathbf{P b}$ en muestras de diésel por GF AAS. Priscila de Oliveira Vicentino, Ricardo Jorgensen Cassella, Martín Resano Ezcaray

- Monitorización de nanopartículas de oro mediante espectroscopia de absorción atómica de fuente continua de alta resolución con atomización electrotérmica. Raúl Garde, Martín Resano, Esperanza García

- Cinética de reacción de hollín de referencia diésel (SRM 1650b - NIST) con NO2 en condiciones del filtro de particulas diésel (DPF). María Abián, Ricardo Pernía, Ángela Millera, Rafael Bilbao, María U. Alzueta

- Purificación de hidrógeno a partir de gas de síntesis mediante el proceso "Steam-Iron". Javier Pérez-Cortés, Paúl Durán, Javier Herguido, José Ángel Peña 
- Caracterización de líquidos de pirólisis para su posterior tratamiento mediante separación o hidrotratamiento. María Atienza-Martínez, Isabel Fonts, Jesús Salafranca, Óscar de la Iglesia, José Luis Sánchez

- Caracterización de la fase acuosa obtenida en la pirólisis de la fracción sólida digerida procedente de purines. Nadia Ruiz-Gómez, María Atienza-Martínez, Javier Ábrego, Fréderic Marías, Gloria Gea

- Efecto de una etapa de pretratamiento con ácido sobre la pirólisis de purines. Nadia Ruiz-Gómez, Cristina Pina, María Atienza-Martínez, Isabel Fonts, Gloria Gea

- Optimización y comparación entre la micro-extracción en fase líquida y la micro-extracción en fase sólida para la determinación de isoftalaldehido en muestras de migración acuosa. Jazmín Osorio, Sara Úbeda, Margarita Aznar, Cristina Nerín

- Identificación y cuantificación de compuestos procedentes de un material multicapa para envase alimentario y estudios cinéticos de migración. Sara Úbeda, Marga Aznar, Paula Vera, Cristina Nerín, Luis Henríquez, Claudia Restrepo

\section{TECNOLOGÍAS DE LA INFORMACIÓN Y LAS COMUNICACIONES}

- Gestión colaborativa de modelos de ciudades 3D durante su ciclo de vida basadas en servicios en la nube. Iñaki Prieto, Jose Luis Izkara, Rubén Béjar

- Design of a New Cryptosystem Combining a MEMS-Accelerometer and a Chaotic Map. Miguel García-Bosque, Adrián Pérez. Carlos Sánchez-Azqueta, Santiago Celma

- Linealización mediante Predistorsión Digital de un Sistema Radio sobre Fibra de Doble Banda. Carlos Mateo, Nieves Pérez, Paloma García, Pedro L. Carro, Jesús de Mingo and ínigo Salinas

- Desarrollando agentes EBDI. Yanet Sánchez, Eva Cerezo

- WCET con cache de instrucciones bloqueable y Lock-MS. Alba Pedro-Zapater, Clemente Rodríguez, Juan Segarra, Rubén Gran, Víctor Viñals-Yúfera

- Análisis de procesos de coalescencia en ferrofluidos mediante holografía digital. Ángel SanzFelipe, Julia Lobera, Juan Carlos Martín, Virginia Palero

- Mejora de un modelo de representación de materiales para su uso en edición intuitiva de apariencia. Sandra Malpica, Miguel Barrio, Diego Gutiérrez, Ana Serrano, Belén Masiá

- RF Transimpedance Amplifier for CATV Applications over PON. Guillermo Royo, Carlos SánchezAzqueta, Concepción Aldea and Santiago Celma

- Approaching Ideal Polyphase Filter Response in 65-nm CMOS. Antonio D. Martínez Pérez, Jorge Morte, Francisco Aznar, Carlos Sánchez Azqueta, Santiago Celma

\section{TECNOLOGÍAS INDUSTRIALES}

- Comparación de ACV de un refuerzo estructural de plancha de acero y de un refuerzo de lámina de fibra de carbono ambos adheridos con epoxi. B. Palacios-Muñoz, B. López-Mesa, L. Gracia-Villa

- Análisis de la capacidad de medición de una máquina herramienta usando el método Monte Carlo para estimar la incertidumbre. Pablo Pérez, Sergio Aguado, José Antonio Albajez, Jorge Santolaria

- A Low-quiescent Current Full on-chip 1.2 V CMOS Low Drop-Out Regulator. Jorge Pérez-Bailón, Belén Calvo, Nicolás Medrano

- A Digitally Programmable Active-RC Filter for On-Chip Portable Sensor Applications. Alejandro Márquez, Nicolás Medrano, Belén Calvo, Pedro A. Martínez

- Síntesis general de arquitecturas de bloques para sistemas de almacenamiento de energía híbridos. Iván Sanz, Carlos Bernal, Estanis Oyarbide

- Métodos de caracterización modal de guías de onda multinúcleo. David Benedicto, Maria Victoria Collados, Juan Carlos Martín, Jesús Atencia, Juan Antonio Vallés

- Lens-Photovoltaic Cell Coupling in a Holographic Solar Concentration System. Julia Marín-Sáez, María Victoria Collados, Daniel Chemisana, Jesús Atencia

- Study of Passive CMOS Mixers Effects on Image Rejection Ratio. Jorge Morte, Antonio D. Martínez Pérez, Francisco Aznar, Carlos Sánchez Azqueta, Santiago Celma 